\title{
EUS guided fine needle aspiration is useful in diagnostic evaluation of indeterminate proximal biliary strictures
}

\author{
Naveen B. Krishna, Pavan Tummala, Jennifer L. Labundy, Banke Agarwal \\ Division of Gastroenterology and Hepatology, Saint Louis University School of Medicine, Saint Louis, USA \\ Email: agarwalb@slu.edu
}

Received 28 February 2012; revised 5 March 2012; accepted 9 April 2012

\begin{abstract}
Background and Aim: Determination of the etiology of proximal biliary strictures (PBS) still remains a challenge. Even though EUS-FNA is potentially useful, its role in diagnostic evaluation of PBS is still not established due to a lack of sufficient data. We sought to determine the performance characteristics of EUSFNA in patients with obstructive jaundice (ObJ) and PBS. Patients and Methods: This is a retrospective analysis and included patients with ObJ and PBS with non-diagnostic brush cytology, who underwent EUS-FNA in our university based practice from March 2002 to February 2009. We evaluated the final diagnoses in study patients and the performance characteristics of EUS-FNA for identifying malignant PBS. Final diagnosis was based on surgical pathology or clinical follow-up of at least 12 months. Results: The study included 28 patients (17 male, 11 female) with mean age of $62.4 \pm 14.9$ years. The strictures ranged from $10-70 \mathrm{~mm}$ in length. CT scan identified a hilar mass in 9 patients. EUS-FNA identified malignancy in 12 patients. 18 patients were finally diagnosed to have a malignant stricture and 10 patients had a benign stricture. There were 6 false negative diagnoses. The accuracy of EUS-FNA for diagnosis of cholangiocarcinoma was $78.6 \%$, with $66.6 \%$ sensitivity, $100 \%$ specificity, $62.5 \%$ NPV and $100 \%$ PPV. Conclusions: EUS-FNA can diagnose malignancy in 66.6\% of jaundiced patients with PBS and non-diagnostic brush cytology and may have a role in their further diagnostic evaluation.
\end{abstract}

Keywords: EUS-FNA; Cholangiocarcinoma; Proximal Biliary Strictures

\section{INTRODUCTION}

EUS-FNA can potentially be useful in patients with pro-

"Conflicts of interest: The authors have no potential conflicts of interest. ximal biliary strictures (PBS) to obtain tissue diagnosis, especially when the brush cytology of the stricture is not diagnostic for malignancy. However, EUS-FNA is still not widely used in the diagnostic evaluation of patients with a PBS, largely due to a lack of sufficient data. Although there are a few reports on the potential role of EUS-FNA for obtaining tissue diagnosis in PBS, the aggregate number of patients in these reports is small [15]. This is probably because PBS are less common than distal strictures, and brush cytology is relatively more sensitive for diagnosing cholangiocarcinoma which is responsible for the vast majority of the PBS [6]. However, there is, still a significant number of patients with PBS, in whom the brush cytology is non-diagnostic, and these patients need to undergo exploratory surgery for determination of etiology and therapy. A definitive pre-operative determination of malignancy in patients with PBS would help in treatment planning and could potentially obviate the need for exploratory surgery in several patients.

We performed a retrospective analysis of performance characteristics of EUS-FNA in our cohort of patients who had PBS, had obstructive jaundice (ObJ) at presentation, and in whom the brush cytology was not diagnostic for malignancy.

\section{PATIENTS AND METHODS}

\subsection{Study Design and Patient Selection}

This represents an analysis of patients from our database of patients who undergo EUS/EUS-FNA at Saint Louis University School of Medicine. The database was started in March 2002 and is maintained prospectively. Followup information is rigorously collected for quality assurance in our clinical practice. It comprises periodic phone calls to patients, in addition to monitoring correspondence from the referring physicians and primary care physicians, operative notes, surgical pathology and imaging reports.

All patients who underwent EUS/EUS-FNA between March 2002 and February 2009 for evaluation of a PBS noted during ERCP performed for $\mathrm{ObJ}$ were considered for inclusion. Patients with brush cytology of the stricture 
that was positive for malignancy were excluded (these patients underwent EUS/EUS-FNA for staging of the bile duct cancer and not for diagnosis). Patients were also excluded if the stricture was confined above the level of hepatic bifurcation. Patients with a history of liver transplantation were not included, as a vast majority of biliary strictures in these patients are benign and have a markedly lower likelihood of malignancy.

The study was approved by the Institutional Review Board of the Saint Louis University School of Medicine.

\subsection{EUS Examination}

EUS examination was performed using radial echoendoscope (EUM-130 or EUM-160 Olympus, Melville, NY). Whenever a suspicious "mass" lesion or area of thickening of the bile duct was identified on radial EUS, FNA was performed using linear echoendoscopes (FG-32A or EG-3870UTK Pentax, Orangeburg, NY). The technique for EUS/EUS-FNA was similar to the one used in previously published studies [1,2]. Multiple FNA passes [1-7] were made using the $22 \mathrm{G}$ or $25 \mathrm{G}$ Echo-tip FNA needle (Wilson Cook, Winston-Salem, NC) until the cytologist could make a preliminary diagnosis. The cytology specimens were stained by the Diff-Quik and Papanicoulou method (Pap smears) and assessed immediately by a cytologist. A sample was also collected for cell block. The final diagnosis was based on examination of the Pap smears and the cell block using standard cytologic criteria [7]. Special cytology stains were used as indicated.

\subsection{Follow Up and Final Diagnosis}

There were no procedure related complications. Final diagnosis was based on definitive cytology, surgical pathology and clinical follow-up of $\geq 12$ months. The median follow-up was 22 months. Cytology that was "atypical" $(n=4)$ or "suspicious" $(n=1)$ for malignancy was counted as negative for malignancy for purposes of this study.

\section{RESULTS}

\subsection{Patient Characteristics}

Thirty patients qualified for inclusion in this study. One patient died of unrelated causes within 1 month after EUS-FNA, did not have an autopsy, was excluded from further analysis due to lack of a definitive final diagnosis and another patient was lost to follow-up. The characteristics of the patients included in the study are summarized in Table 1. In the twenty-eight patients included (17 male, 11 female), the mean age was $62.4 \pm 14.9$ years (range 23 - 87). All patients had ObJ at initial presentation and had undergone an ERCP with placement of a plastic biliary stent prior to EUS-FNA. Three
Table 1. Patient Characteristics.

\begin{tabular}{lc}
\hline & $\mathbf{N}=\mathbf{2 8}(\mathbf{\%})$ \\
\hline Age (years) & $62.4 \pm 14.9(23-87)$ \\
Gender & $17(60.7)$ \\
$\quad$ Male & $11(39.3)$ \\
$\quad$ Female & $10-70 \mathrm{~mm}$ \\
Stricture length (range) & \\
CT findings & $9(32.1)$ \\
$\quad$ Mass at liver hilum & $2(7.1)$ \\
$\quad$ Enlarged periportal LN & \\
Benign/Malignant on final diagnosis & $10(35.7)$ \\
$\quad$ Benign & $18(64.3)$ \\
$\quad$ Malignant & \\
Status of the patient & $15(53.6)$ \\
$\quad$ Dead & $13(46.4)$ \\
$\quad$ Alive & $22(14-37)$ \\
\hline
\end{tabular}

patients had cholangiographic evidence of Primary sclerosing cholangitis (PSC) in the intra-hepatic biliary tree and had a dominant stricture at the hilum with suspicion for cancer. In addition to the dilation of the intrahepatic biliary tree on CT scans, in nine patients there was an identifiable mass lesion at or near the liver hilum, and in another two patients, enlarged periportal lymph nodes were noted. The length of the biliary stricture ranged from $10-70 \mathrm{~mm}$. Brush cytology from the stricture was non-diagnostic or negative for malignancy in all patients.

\subsection{EUS Findings}

EUS examination revealed thickening of the bile duct wall or a mass lesion in twenty-five of the twenty eight patients. The size of the hilar lesion ranged from $15-45$ $\mathrm{mm}$. Enlarged periportal lymph nodes were noted by EUS in sixteen patients and measured $10-30 \mathrm{~mm}$ in size. FNA of the enlarged periportal lymph nodes revealed metastatic adenocarcinoma in three patients. FNA of the hilar lesion/biliary stricture identified malignant cells in twelve patients. In one patient, the cells were considered suspicious for malignancy; in four patients they were reported as atypical but not diagnostic for malignancy, in eleven patients there were no cytologic evidence of malignancy.

\subsection{Final Diagnoses and Performance Characteristics of EUS-FNA}

The diagnosis based on EUS-FNA, follow-up and final diagnoses in study patients are summarized in Figure 1. Surgical consult was obtained in all patients for consi- 


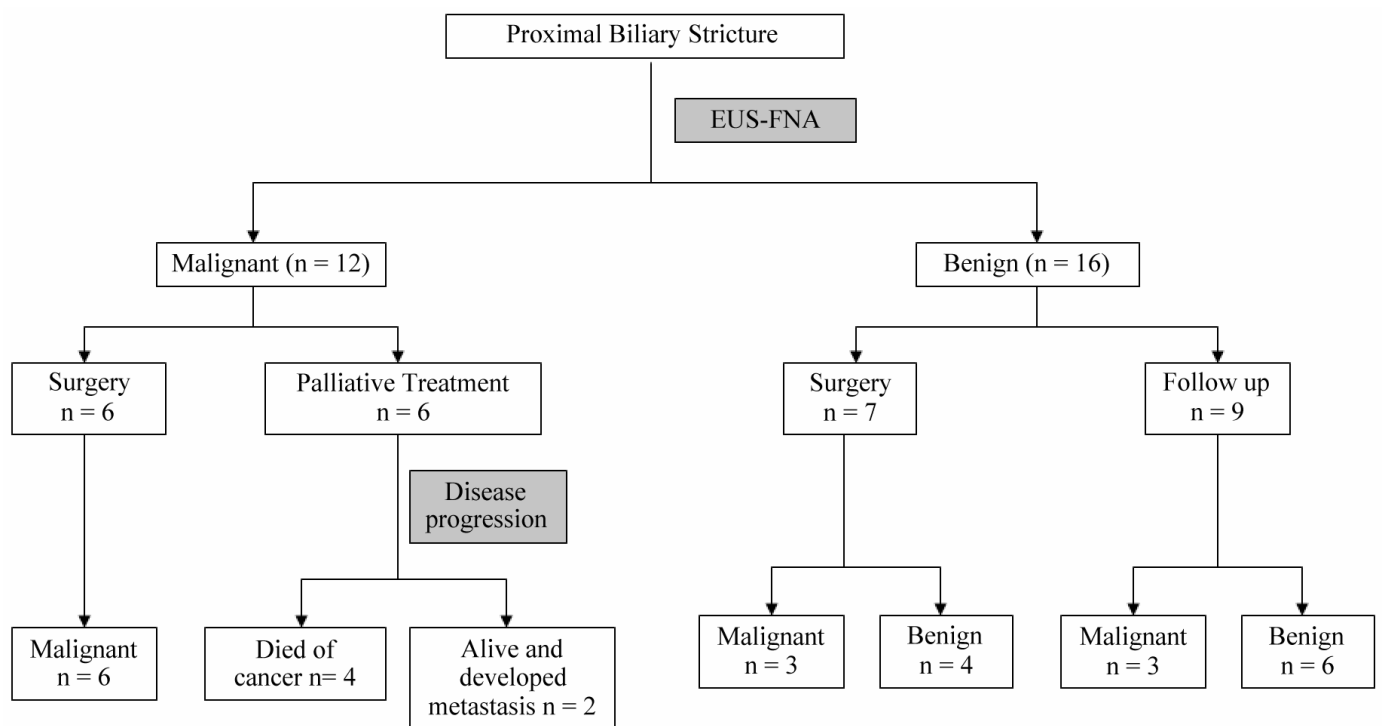

Figure 1. Summary of diagnosis based on EUS-FNA, follow-up and the final diagnosis in study patients.

deration for surgery. Thirteen of the twenty-eight study patients underwent surgery: including six patients diagnosed to have a malignant stricture and seven patients diagnosed to have a benign stricture by EUS-FNA.

The diagnosis of malignancy was confirmed by surgery in all six patients diagnosed by EUS-FNA to have malignant stricture: two of these patient were found to unresectable tumors during surgery and in the remaining four patients only one patient had a R0 resection. Six patients diagnosed to have a malignant stricture by EUSFNA did not undergo surgery because of locally advanced or metastatic cancer. Four of these patients died of cancer and two of them were alive at time of follow up but had developed new metastatic lesions.

Of the seven patients diagnosed to have benign stricture by EUS-FNA who underwent surgery, three were found to have malignant strictures due to cholangiocarcinoma. Two of these three patients were considered to have resectable tumor during surgery; however R0 resection was achieved in neither of these patients. Two of four patients confirmed to have a benign stricture by surgery had inflammatory pseudo-tumors, one had primary sclerosing cholangitis and the etiology was unclear in the fourth patient. Nine patients diagnosed to have a benign stricture by EUS-FNA, did not undergo surgery either because of co-morbid conditions or because they chose not to have surgery. Three of these patients were later diagnosed to have a malignant biliary stricture and died of cancer. The remaining six patients continue to do well 12 to 26 months after EUS-FNA. The probable etiology of the benign stricture in these patients was: primary sclerosing cholangitis in two patients based on suggestive changes in the intrahepatic biliary tree and the etiology was unclear in the remaining four patients.
There were six false negative diagnoses: three patients had cancer detected by surgery, and three patients had disease progression with metastasis. All the six patients died of cancer. Cytologic diagnoses based on FNA specimens from the bile duct stricture of these patients included "suspicious for adenocarcinoma" $(\mathrm{n}=1)$, "atypical" $(n=3)$ and "benign ductal cells" $(n=2)$. There were no false positive diagnoses amongst study patients. Performance characteristics of EUS-FNA for diagnosing malignancy in the proximal biliary stricture in the study patients are summarized in Table 2.

\section{DISCUSSION}

It is helpful to be able to determine the presence or

Table 2. Performance characteristics of EUS-FNA for malignancy.

\begin{tabular}{|c|c|c|}
\hline & \multicolumn{2}{|c|}{$\mathbf{N}=\mathbf{2 8}$} \\
\hline True Positive & & \\
\hline True negative & & \\
\hline False negative & & \\
\hline Sensitivity (95\% CI) & $12 / 18$ & $\begin{array}{c}66.6 \% \\
(41.1,85.6)\end{array}$ \\
\hline Specificity & $10 / 10$ & $\begin{array}{c}100 \% \\
(65.5,100)\end{array}$ \\
\hline Negative predictive value & $10 / 16$ & $\begin{array}{c}62.5 \% \\
(35.8,83.7)\end{array}$ \\
\hline Positive predictive value & $12 / 12$ & $\begin{array}{c}100 \% \\
(69.8,100)\end{array}$ \\
\hline Accuracy & $22 / 28$ & $\begin{array}{c}78.6 \% \\
(63.3,93.7)\end{array}$ \\
\hline
\end{tabular}


absence of malignancy in biliary strictures to individualize and optimize further management of these patients. This is now becoming even more important due to the availability of alternative treatment options for these patients [8-14]. However identification of malignant biliary strictures is not always easy and largely depends on brush cytology of the stricture. Biliary brush cytology is notoriously unreliable for the diagnosis of malignancy, though its sensitivity is slightly better for diagnosing cholangiocarcinoma than for pancreatic adenocarcinoma [15]. Efforts to improve the pre-operative identification of malignancy in biliary strictures using better sampling methods [16-19] or using novel markers [20-24] have resulted in only a modest improvement in the diagnostic yield. There is, therefore, a need for new diagnostic methods to increase the proportion of patients in whom a reliable pre-operative diagnosis of cancer can be achieved.

In the past decade, use of EUS-FNA has significantly improved the pre-operative determination of malignancy in patients with a distal biliary stricture with reported sensitivity of $73 \%-90 \%$ [25-31] for the diagnosis of pancreatic cancer, which accounts for the vast majority of malignant distal biliary strictures. However, EUS-FNA is still not widely used in patients with PBS. There is a need for more data on the sensitivity and accuracy of EUS-FNA for identifying malignancy in PBS, as the currently available data is limited by the relatively small number of published studies and the small aggregate number of patients in these studies [1-5]. This is probably because PBS are less common than distal biliary strictures, and the sensitivity of brush cytology is higher for diagnosing cholangiocarcinoma. In addition, we believe that only a fraction of eligible patients with indeterminate proximal biliary stricture are referred for EUS-FNA for tissue diagnosis due to a lack of awareness about its potential use for this indication amongst the referring physicians, and probably also because it is technically more challenging $[32,33]$ and is not routinely performed in several centers. The number of patients in the present study is also modest, even though ours is a high volume center for endoscopic ultrasound and these patients were accrued over a period of about seven years. The number of patients is however comparable to those from another reputed large volume center in the Unites States over a similar period of time [1].

Patients with PBS are usually taken for exploratory surgery, when the brush cytology of the stricture is negative for malignancy or is non-diagnostic. Some authors have argued that all patients with PBS be taken for surgery because $90 \%$ of these strictures are malignant and with currently available tests, it is not possible to reliably exclude malignancy [34]. However this study comprised patients from a major stand alone cancer center and probably had a recruitment bias based on referral patterns unique to such a center. In our cohort from university based practice, only 18 of 28 patients $(64 \%, 95 \% \mathrm{CI}-46.5,82.0)$ had malignant strictures. Moreover potentially curative resection is possible only in a small number of patients with cholangiocarcinoma. Exploratory surgery can potentially be avoided in patients with unresectable tumor based on radiologic imaging by availability of a pre-operative diagnosis of malignancy. EUS-FNA, by providing tissue diagnosis, may obviate the need for surgery in a significant proportion of these patients. EUS-FNA may also help identify and sample the enlarged periportal and celiac lymph nodes that might determine resectability and help in treatment planning.

Management of patients with ObJ and PBS is often a challenge for fear of missing an opportunity to treat a potentially resectable neoplasm. Figure 2 summarizes our recommended approach to diagnosis and management of proximal biliary strictures brush cytology, EUS-FNA and Intraductal ultrasound (IDUS). The yield of brush cytology and EUS-FNA can potentially also be increased by testing the tissue samples by novel methods including flourescent in situ hybridization (FISH) and digital image analysis (DIA). If a diagnosis of malignancy still cannot be achieved by the above-mentioned methods, IDUS may be helpful. We have earlier reported that the bile duct wall thickness at stricture site $\leq 7 \mathrm{~mm}$ strongly suggests a benign biliary stricture whereas wall thickness $\geq 8 \mathrm{~mm}$ was strongly associated with malignant strictures [35]. Similar observations were made in two earlier published studies [36,37]. After biliary brush cytology and EUS-FNA have failed to identify malignancy in proximal biliary strictures, patients with bile duct wall thickness $\geq 8 \mathrm{~mm}$ may be taken for exploratory surgery because of higher likelihood of malignancy. On the other hand, patients with bile duct wall thickness $<8 \mathrm{~mm}$ may be managed conservatively with repeat imaging and sampling in $6-12$ weeks and endoscopic management of these strictures.

In this study, we excluded patients with strictures confined to the intrahepatic ducts. This is because in our experience it is often difficult to identify cholangiocarcinoma within the liver with EUS as it can be isodense with the liver parenchyma. Our bias is that EUS-FNA is probably not as useful for evaluation of a stricture confined to intrahepatic ducts as it is for extrahepatic biliary strictures. Secondly, we included only those patients who had ObJ at initial presentation because only in this group, routine surgical exploration is recommended in absence of tissue diagnosis due to a very high likelihood of malignancy. Even though PBS in patients without ObJ can have a malignant etiology [1], the likelihood of malignancy is much less than in patients with $\mathrm{ObJ}$ and routine surgical exploration is currently not recommended in these patients. Similarly, we also ex- 


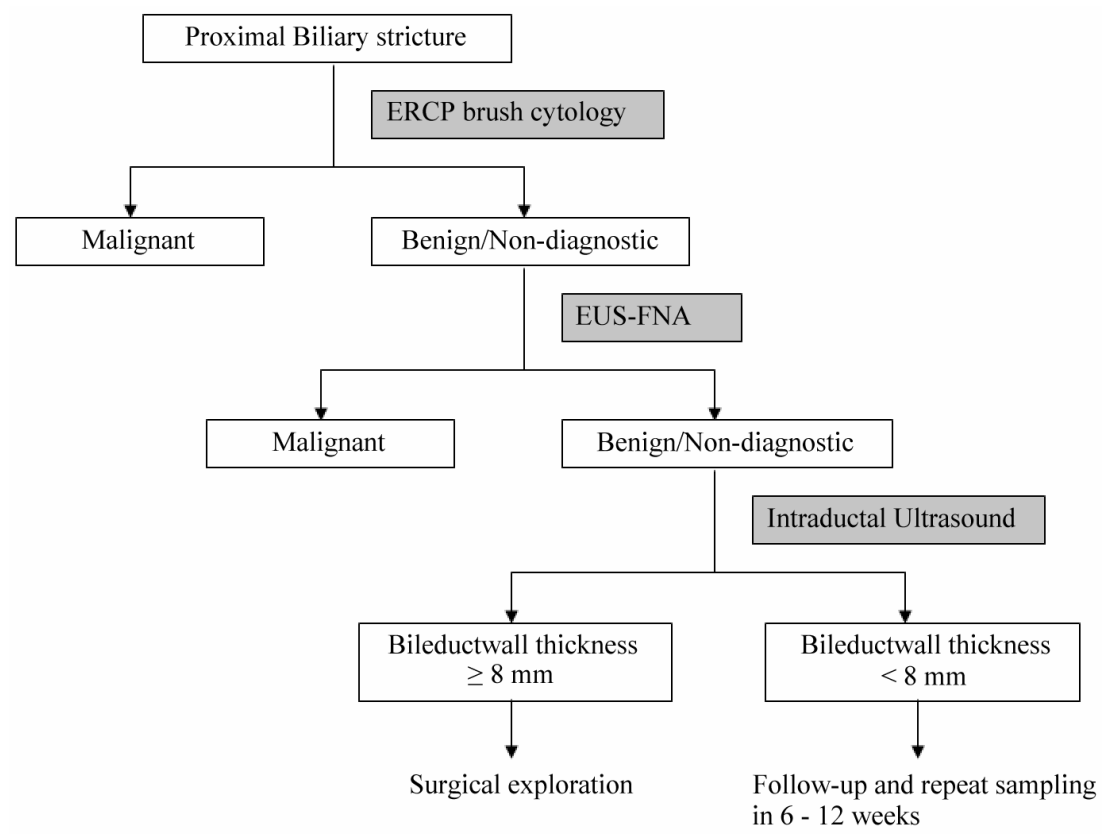

Figure 2. Suggested algorithm for endoscopic evaluation of patients with obstructive jaundice and a proximal biliary stricture.

cluded patients with history of liver transplantation because the vast majority of biliary strictures in these patients are benign. In some centers orthotopic liver transplantation is performed for potentially resectable cholangiocarcinoma and EUS-FNA of the stricture is considered a contraindication in these patients for fear for local tumor spread. However, only a very small fraction of extrahepatic cholangiocarcinomas are potentially resectable. In our cohort, only 6 of 18 patients with cholangiocarcinoma finally underwent surgical resection and only 1 patient had R 0 resection. It might therefore be reasonable to perform cross-sectional imaging to determine resectability of suspected tumor prior to performing EUS-FNA. If there is sufficient adjunctive clinical data to support a diagnosis of cholangiocarcinoma and the patient is a candidate for potentially curative surgery, then EUS should be performed only to look for malignant lymphadenopathy that would preclude liver transplantation. If however there is radiologic evidence of unresectability or the patient is not a good surgical candidate, EUS-FNA for tissue diagnosis is probably a reasonable alternative to exploratory surgery.

The present study has limitations inherent to its retrospective design and our findings are therefore not definitive. However the study is based on data from our prospective database and final diagnosis was available in 28 of 30 patients who met the inclusion criteria for this study. In addition, the number of patients is small like other previously published studies for reasons mentioned earlier. Another potential limitation is that all the patients in this study had a biliary stent in place at the time of
EUS examination. Presence of a biliary stent can potentially help in identifying the biliary stricture as the bile duct is followed towards the hilum of liver with the echoendoscope. However, presence of biliary stent also induces inflammation which can cause cellular atypia mimicking well differentiated adenocarcinoma. This makes cytologic interpretation of EUS-FNA specimens difficult and can potentially lower the sensitivity of EUS-FNA. Despite these limitations, our data adds to the earlier published data and supports the potential use of EUSFNA in the diagnostic evaluation of PBS.

To conclude, we found that in patients with $\mathrm{ObJ}$ who are found to have a PBS on ERCP with non-diagnostic brush cytology of the stricture can provide tissue diagnosis in $66.6 \%(95 \%$ CI $41.8,88.4)$ of patients. EUS-FNA may have a role in selected patients with indeterminate PBS to minimize the need for exploratory surgery.

\section{HIGHLIGHTS}

\subsection{What Is Current Knowledge?}

The vast majority of proximal biliary strictures in patients with obstructive jaundice are malignant.

Pre-operative diagnostic testing to determine etiology is of limited value when biliary brush cytology is nondiagnostic.

\subsection{What Is New Here?}

The prevalence of malignancy in above-defined patients is probably much lower than reported earlier. 
EUS-FNA can provide tissue diagnosis in significant proportion of these patients and avoid exploratory surgery for diagnosis.

\section{REFERENCES}

[1] DeWitt, J., Misra, V.L., Leblanc, J.K., et al. (2006) EUS-guided FNA of proximal biliary strictures after negative ERCP brush cytology results. Gastrointestinal Endoscopy, 64, 325-333. doi:10.1016/j.gie.2005.11.064

[2] Fritscher-Ravens, A., Broering, D.C., Knoefel, W.T., et al. (2004) EUS-guided fine-needle aspiration of suspected hilar cholangiocarcinoma in potentially operable patients with negative brush cytology. The American Journal of Gastroenterology, 99, 45-51.

doi:10.1046/j.1572-0241.2003.04006.x

[3] Fritscher-Ravens, A., Broering, D.C., Sriram, P.V., et al. (2000) EUS-guided fine-needle aspiration cytodiagnosis of hilar cholangiocarcinoma: A case series. Gastrointestinal Endoscopy, 52, 534-540. doi:10.1067/mge.2000.109589

[4] Rösch, T., Hofrichter, K., Frimberger, E., et al. (2004) ERCP or EUS for tissue diagnosis of biliary strictures? A prospective comparative study. Gastrointestinal Endoscopy, 60, 390-396. doi:10.1016/S0016-5107(04)01732-8

[5] Eloubeidi, M.A., Chen, V.K., Jhala, N.C., et al. (2004) Endoscopic ultrasound-guided fine needle aspiration biopsy of suspected cholangiocarcinoma. Clinical Gastroenterology Hepatology, 2, 209-213. doi:10.1016/S1542-3565(04)00005-9

[6] Glasbrenner, B., Ardan, M., Boeck, W., et al. (1999) Prospective evaluation of brush cytology of biliary strictures during endoscopic retrograde cholangiopancreatography. Endoscopy, 31, 712-717. doi:10.1055/s-1999-73

[7] Lin, F. and Staerkel, G. (2003) Cytologic criteria for well differentiated adenocarcinoma of the pancreas in fineneedle aspiration biopsy specimens. Cancer, 99, 44-50. doi:10.1002/cncr.11012

[8] Chahal, P. and Baron, T.H. (2006) Endoscopic palliation of cholangiocarcinoma. Current Opinion in Gastroenterology, 22, 551-560.

doi:10.1097/01.mog.0000239872.12081.a4

[9] Simmons, D.T., Baron, T.H., Petersen, B.T., et al. (2006) A novel endoscopic approach to brachytherapy in the management of Hilar cholangiocarcinoma. The American Journal of Gastroenterology, 101, 1792-1796. doi:10.1111/j.1572-0241.2006.00700.x

[10] Malhi, H. and Gores, G.J. (2006) Review article: The modern diagnosis and therapy of cholangiocarcinoma. Alimentary Pharmacology \& Therapeutics, 23, 1287-1296. doi:10.1111/j.1365-2036.2006.02900.x

[11] Nagino, M., Kamiya, J., Nishio, H., et al. (2006) Two hundred forty consecutive portal vein embolizations before extended hepatectomy for biliary cancer: Surgical outcome and long-term follow-up. Annals of Surgery, 243, 364-372. doi:10.1097/01.sla.0000201482.11876.14

[12] Arnagin, W.R., Zager, J.S., Hezel, M., et al. (2006)
Treatment of cholangiocarcinoma with oncolytic herpes simplex virus combined with external beam radiation therapy. Cancer Gene Therapy, 13, 326-334.

[13] Rea, D.J., Heimbach, J.K., Rosen, C.B., et al. (2005) Liver transplantation with neoadjuvant chemoradiation is more effective than resection for hilar cholangiocarcinoma. Annals of Surgery, 242, 451-458.

[14] Ortner, M.E., Caca, K., Berr, F., et al. (2003) Successful photodynamic therapy for nonresectable cholangiocarcinoma: A randomized prospective study. Gastroenterology, 125, 1355-1363. doi:10.1016/j.gastro.2003.07.015

[15] Mansfield, J.C., Griffin, S.M., Wadehra, V., et al. (1997) A prospective evaluation of cytology from biliary strictures. Gut, 40, 671-677.

[16] Harewood, G.C., Baron, T.H., Stadheim, L.M., et al. (2004) Prospective, blinded assessment of factors influencing the accuracy of biliary cytology interpretation. The American Journal of Gastroenterology, 99, 14641469. doi:10.1111/j.1572-0241.2004.30845.x

[17] De Bellis, M., Fogel, E.L., Sherman, S., et al. (2003) Influence of stricture dilation and repeat brushing on the cancer detection rate of brush cytology in the evaluation of malignant biliary obstruction. Gastrointestinal Endoscopy, 58, 176-182. doi:10.1067/mge.2003.345

[18] Farrell, R.J., Jain, A.K., Brandwein, S.L., et al. (2001) The combination of stricture dilation, endoscopic needle aspiration, and biliary brushings significantly improves diagnostic yield from malignant bile duct strictures. Gastrointestinal Endoscopy, 54, 587-594. doi:10.1067/mge.2001.118715

[19] Venu, R.P., Geenen, J.E., Kini, M., et al. (1990) Endoscopic retrograde brush cytology. A new technique. Gastroenterology, 99, 1475-1479.

[20] Van Heek, N.T., Clayton, S.J., Sturm, P.D., et al. (2005) Comparison of the novel quantitative ARMS assay and an enriched PCR-ASO assay for K-ras mutations with conventional cytology on endobiliary brush cytology from 312 consecutive extrahepatic biliary stenoses. Journal of Clinical Pathology, 58, 1315-1320. doi:10.1136/jcp.2004.022707

[21] Khalid, A., Pal, R., Sasatomi, E., et al. (2004) Use of microsatellite marker loss of heterozygosity in accurate diagnosis of pancreaticobiliary malignancy from brush cytology samples. Gut, 53, 1860-1865. doi:10.1136/gut.2004.039784

[22] Kipp, B.R., Stadheim, L.M., Halling, S.A., et al. (2004) A comparison of routine cytology and fluorescence in situ hybridization for the detection of malignant bile duct strictures. The American Journal of Gastroenterology, 99, 1675-1681. doi:10.1111/j.1572-0241.2004.30281.x

[23] Baron, T.H., Harewood, G.C., Rumalla, A., et al. (2004) A prospective comparison of digital image analysis and routine cytology for the identification of malignancy in biliary tract strictures. Clinical Gastroenterology and Hepatology, 2, 214-219. doi:10.1016/S1542-3565(04)00006-0

[24] Tascilar, M., Sturm, P.D., Caspers, E., et al. (1999) Diagnostic p53 immunostaining of endobiliary brush cytology: Preoperative cytology compared with the surgical 
specimen. Cancer, 87, 306-311. doi:10.1002/(SICI)1097-0142(19991025)87:5<306::AIDCNCR11>3.0.CO;2-Y

[25] Agarwal, B., Abu-Hamda, E., Molke, K.L., et al. (2004) Endoscopic ultrasound-guided fine needle aspiration and multidetector spiral CT in the diagnosis of pancreatic cancer. The American Journal of Gastroenterology, 99, 844-850. doi:10.1111/j.1572-0241.2004.04177.x

[26] Chang, K.J., Nguyen, P., Erickson, R.A., et al. (1997) The clinical utility of endoscopic ultrasound-guided fineneedle aspiration in the diagnosis and staging of pancreatic carcinoma. Gastrointestinal Endoscopy, 45, 387-393. doi:10.1016/S0016-5107(97)70149-4

[27] Giovannini, M., Seitz, J.F., Monges, G., et al. (1995) Fine-needle aspiration cytology guided by endoscopic ultrasonography: Results in 141 patients. Endoscopy, 27, 171-177. doi:10.1055/s-2007-1005657

[28] Wiersema, M.J., Vilmann, P., Giovannini, M., et al. (1997) Endosonography-guided fine-needle aspiration biopsy: Diagnostic accuracy and complication assessment. Gastroenterology, 112, 1087-1095. doi:10.1016/S0016-5085(97)70164-1

[29] Faigel, D.O., Ginsberg, G.G., Bentz, J.S., et al. (1997) Endoscopic ultrasound-guided real-time fine-needle aspiration biopsy of the pancreas in cancer patients with pancreatic lesions. Journal of Clinical Oncology, 15, 14391443.

[30] Williams, D.B., Sahai, A.V., Aabakken, L., et al. (1999) Endoscopic ultrasound guided fine needle aspiration biopsy: A large single centre experience. Gut, 44, 720-726. doi:10.1136/gut.44.5.720

[31] Fritscher-Ravens, A., Izbicki, J.R., Sriram, P.V., et al.
(2000) Endosonography-guided, fine-needle aspiration cytology extending the indication for organ-preserving pancreatic surgery. The American Journal of Gastroenterology, 95, 2255-2260. doi:10.1111/j.1572-0241.2000.02311.x

[32] Tio, T.L. and Tytgat, G.N. (1986) Endoscopic ultrasonography of bile duct malignancy and the preoperative assessment of local resectability. Scandinavian Journal of Gastroenterology, 123, 151-157. doi: $10.3109 / 00365528609091877$

[33] Vilmann, P. (1998) Endoscopic ultrasound-guided fineneedle biopsy in Europe. Endoscopy, 30, 161-162. doi:10.1055/s-2007-1001506

[34] Corvera, C.U., Blumgart, L.H., Darvishian, F., et al. (2005) Clinical and pathologic features of proximal biliary strictures masquerading as hilar cholangiocarcinoma. Journal of the American College of Surgeons, 201, 862869. doi:10.1016/j.jamcollsurg.2005.07.011

[35] Krishna, N.B., Saripalli, S., Safdar, R., et al. (2007) Intraductal US in evaluation of biliary strictures without a mass lesion on CT scan or magnetic resonance imaging: Significance of focal wall thickening and extrinsic compression at the stricture site. Gastrointestinal Endoscopy, 66, 90-96. doi:10.1016/j.gie.2006.10.020

[36] Tamada, K. and Sugano, K. (2000) Diagnosis and non-surgical treatment of bile duct carcinoma: Developments in the past decade. Journal of Gastroenterology, 35, 319-325. doi:10.1007/s005350050355

[37] Tamada, K., Tomiyama. T., Wada, S., et al. (1999) Detection of bile duct cancer in an early stage by intraductal ultrasonography (in Japanese with English abstract). Endoscopy, 11, 1164-1172. 